\title{
"Serious Adverse Events" Faced by Clinical Trials: Impact of the COVID-19 Pandemic
}

The ongoing COVID-19 (SARS-CoV-2) pandemic has affected the lives of people in more than 190 countries (almost the entire globe) in a short span of 4 months. ${ }^{[1]}$ As we come to terms with its far reaching implications, there is also the clear and present danger to the 262,366 ongoing randomized clinical trials, as listed on clinicaltrial. gov. ${ }^{[2]}$ This danger is not just in terms of recruitment and immediate patient care, but also for the data collection and analysis to follow-in the medium and long term.

This is a likely scenario because the first priority is to ensure that all health-care professionals are available to tackle the ongoing pandemic instead of being occupied by less important activities - a triage of health-care professional time during these trying times. ${ }^{[3]}$

Priority is also being assigned to research on prevention and/or treatment of COVID-19, particularly the studies of public health importance. This is especially true for those involved in vaccines and other related therapies, from which expectations are high. In fact a PubMed search using "COVID-19" done on March 28, 2020 revealed 1795 references. ${ }^{[4]}$

At the same time, it is our responsibility to give thought to adverse effects on other ongoing clinical trial and how to mitigate them. ${ }^{[5]}$

Fighting the COVID-19 pandemic, in a way, is a step backward with respect to all the rigor that clinical trials require - adherence to timelines and schedules, compliance with guidelines and study protocol, detailed and complete follow-up evaluation, administration of study medication, reporting of symptoms and side effects, finding answers to questions about their conditions. ${ }^{[6]}$ Benefits from treatment is never a given even under standard of care, failure of intended benefit is never assured (often called act of God in legal parlance). ${ }^{[7]}$ For randomized clinical trials, the very purpose is to generate high-quality data on whether there is any benefit or not - an objective that can be answered only after the trial is completed (as per protocol) and the data analyzed.

We cannot think of a better example of how decisions need to be made on a case to case basis.

In the UK, all other new clinical trials have been stopped indefinitely. ${ }^{[3]}$ For ongoing trials, the decision on whether to halt recruitment or not is left in the hands of the local NHS authorities. DHSC issues guidance on the impact of COVID-19 on research funded or supported by NIHR, updated 19 Mar 2020. ${ }^{[8]}$ However, there is the other side of the coin as well. Doctors like Philip Philip GI oncology team lead at Karmanos Cancer Institute, Detroit, Michigan, are of the opinion that, as access to health care for routine checkups takes a beating, some specialists will be able to devote sufficient time to clinical trials, while continuing to play their role against the COVID-19 pandemic. ${ }^{[9]}$

The question facing us, therefore is, how to minimize disruption for patients already recruited in interventional trials ${ }^{[5]}$ Currently, this is not a priority for most health-care facilities or personnel. Whether ongoing clinical trials have the possibility of providing benefit to patients affected by various diseases, is not on the radar right now. The discussion needs to involve all concerned stakeholders, including Clinical Trial Investigators, trial staff, patients, Ethics Committee/Institutional Review Board (EC/IRB), sponsors, CROs, regulatory authorities, ancillary service providers. Understandably, some authorities, institutional ECs, and Clinical Trial Investigators differ significantly on what is the best way forward. Other stakeholders have either not been consulted, have no decision making power or are simply not able fulfill their role under the prevailing conditions. This balancing act requires proactive discussion and agreement between Sponsors, CROs, principal investigators, and EC. When such an opportunity is not forthcoming, the clinical trial investigators would have to simultaneous juggling multiple responsibilities and decide (on their own or in consultation with their research team) at their discretion. This could mean immediate suspension (temporarily) of clinical patient research that does not have the potential to lead to substantial patient benefit (e.g., trials to improve understanding of pharmacokinetics and mechanism of action) versus continuation of enrolled patients on trial medication (e.g., adjuvant therapy to increase chance of cure in cancer patients) where the primary objective is to improve outcome among participants (as proposed by Fred Hutchinson Cancer Research Center last week). ${ }^{[5]}$

Clinical trials and drug development can truly be seen at the cusp of balancing resources in the global health-care ecosystem faced with unprecedented challenges.

How do we decide the right answer for a patient with Stage IV cancer, who has exhausted several lines of standard of care, and is in a clinical trial to receive a novel therapeutic study medication that has the potential to substantially improve overall survival or quality of life (QoL)? What is very clear is that difficult questions need to be asked and each team will need to come up with a strategy to address them. The worst answer (even though it might actually be true) that anyone has to give or hear is "I don't know." 
Someone has to come up with new ideas on how to optimize overall benefit to society as well as make efficient use of resources within the limitations imposed by the pandemic. We will therefore focus the rest of the discussion from the point of view of the Clinical Trial Investigators. We must live up to our commitment to regulatory authorities, EC, sponsors, participants and research staff by reworking and executing measures while also keeping everyone safe from the clutches of COVID-19 pandemic.

An SOP needs to be developed very quickly on what needs to be done - to address one or more of the following:

Communication lines need to be updated for routine and urgent purposes with trial staff, institution authorities (administrative as well as EC), Sponsors and CROs as well as with the drug authority (US Food and Drug Administration [FDA], Indian DCGI or country equivalent). We have previously reported that some investigators with ongoing trials done even know whether their EC is currently functional or not. ${ }^{[10]}$

Information needs to keep flowing - especially regarding any changes in schedule of events, trial medication, investigations, adverse reactions and serious adverse events (SAEs). This will help reassure patients, minimize protocol deviations and secure integrity of data captured. ${ }^{[3,5,10]}$

For patients it is an important decision between following instructions from public health authorities to stay at home in self-isolation family bubbles versus going to the health-care facility for treatment and related clinical trial schedule of events. ${ }^{[11]}$ The former would mean missing out on the potential benefit of trial participation and risking the possibility of disease progression with all its consequences (temporary or permanent). The latter option meant risking entry into an area where there is highest chance of coming into contact with another person who is already affected by the COVID-19 virus and consequences that could be a real danger to life in the immediate future. ${ }^{[12]}$ The choice and uncertainty of reliable information makes the entire experience extremely stressful. This is compounded by the honest answer from their doctors, namely "we do not know enough to tell you which is the better option."

Several health-care professionals, including oncologists are sharing tips on managing specific patient groups in the real world (outside a clinical trial). For example how to deal with immunotherapy and chemotherapy schedules. ${ }^{[13]}$ Such messages have been disseminated widely through social media and community newsletters.

Virtual consultation and examination (akin to verbal autopsy) can replace in-clinic visits. ${ }^{[14,15]}$ Newly issued guidelines from the "Medicines and Healthcare Products Regulatory Agency" now permit replacement of face to face study visits by telephonic consultation. ${ }^{[16]}$ Ongoing trials also need to factor in the vital need to be flexible with schedule of events while facing the COVID-19 pandemic.

The NRG on their website has stated that investigators should explore other means for gathering data, perhaps via teleconsultation and virtual visits. They also give the assurance that such an approach in clinical trials will not be considered as protocol deviation or noncompliance. This is, presumably, temporary during the disruption caused by the COVID-19 pandemic. $^{[17]}$

The FDA's guidelines refer to matters concerning investigational study drug, consent withdrawal by patients, safety monitoring, etc., In all such instances current challenges can be resolved using telephonic conversation and virtual visits. Assessment and investigations can also be done at nontrial approved facilities - including blood tests (like biochemistry) and radiological imaging (like computed tomography scans), especially for safety. ${ }^{[18]}$ Centers for Medicare and Medicaid Services are now approving use of telemedicine technology for Medicare beneficiaries during the pandemic period. For their patient in clinical trial, this will apply only if they are receiving Medicare-covered drug during approved trials. ${ }^{[19]}$

Careful documentation of crucial data points regarding efficacy and safety should be the focus. Online submission of QoL questionnaires can be facilitated by Google forms or specific apps. ${ }^{[12]}$ Attention to confidentiality should be continued while using such temporary measures. Paper copies of self-administered questionnaires can be filled by hand, photographed using smartphones and E-mailed to the study site personnel.

Social distancing has impacted to other in-person meetings as well. US FDA has postponed all routine inspections. Advisory committee (Adcomm) meetings are now virtual and they are taking advantage of technology to have meetings that permit remote participation. Educational medical meetings too have been cancelled, postponed or moved to the online platform (e.g., American College of Cardiology's Annual World Congress and American Society of Clinical Oncology's annual meeting, both usually held in McCormick Place, Chicago. ${ }^{[20]}$

Monitoring is affected to a major extent. For instance, trial monitors are not permitted entry in the premises of the Common Spirit Health hospital system. ${ }^{[20]}$ These activities also need to be postponed or moved to the virtual platform. Those with electronic case record forms are either doing this already or can easily change their procedure appropriately. This will limit the unnecessary risk of being exposed to the COVID-19 virus.

Trial related investigations should be revisited. Some may not be possible at all. Even when feasible, there should be discussion on which ones are important for preserving data integrity, especially from safety and regulatory point of view. Similarly, imaging studies might need to be 
postponed, delayed or even cancelled while the pandemic is galloping.

Trial related/associated medication includes investigational product (IP) as well as medicines that the patient requires both on and off study. If the IP is not be given parenterally, health-care facility visit might be avoidable. Also oral medicines need not be collected by patients themselves. Either healthy caregivers can come to pick them up in a safe environment or the site can plan to have the IP delivered to patients' homes. How to do this safely with limited travel options also needs innovative solutions.

Reporting to ethics committees, sponsors and drug authorities should be continued as originally intended. Any deviation in terms of details or reporting deadlines should be recorded with specific reason for the same.

All stake holders need to update themselves in real time regarding any evidence of study related intervention influencing COVID-19 or vice versa. If such information becomes available, study team members should be extra vigilant regarding the same. Trial sponsors should also share such information with other investigators/sites and with regulatory authorities as appropriate.

When a patient on study is suspected to have COVID-19 symptoms or infection, the decision will be even more difficult. The Clinical Trial Investigators primary objective is the safety of trial subjects. Aggressive management of COVID-19 will be the first priority. This will mean following government directives (health authorities might require mandatory reporting) and may even mean shifting the patient to a COVID-19 specified center. The study protocol will have to be revisited to find out if withdrawal from the study is required or not. Becoming COVID-19 positive will need to be reported as an SAE to EC, Sponsor/ $\mathrm{CRO}$ as well as drug regulatory authorities. All personnel who have come in contact with the patient will need to follow institutional policy in dealing with COVID-19 contacts.

The current lack of widespread COVID-19 will be a significant factor in the eventual reporting of clinical trial results. COVID-19 testing is unlikely to be part of protocols of ongoing trials. How do we deal with this from a regulatory and data integrity point of view? While trials are designed to take into consideration some variations and unknown factors, trial study size will not have been decided to neutralize the impact of sick trial patients with the additional morbidity of having a COVID-19 infection.

It is always good to widen the communication circle. Asking all (old and new) patients to provide at least three additional contact persons (family or friends), and their preferred means of communication (phone, app, E-mail) will be useful. This is especially necessary since it is a real possibility that communication with the study participant may be compromised due to several factors (e.g., interruption to cellular operator signal tower in the vicinity of the individual's home).

The current pandemic is severe enough to be frightening to some. Hence, discussion should be initiated with all trial subjects regarding their right to withdraw consent and to decline specific study related events that they are uncomfortable with. ${ }^{[10]}$ There is sufficient justification to also discuss, and take consent regarding the potential risks of continuing with trial participation while the COVID-19 pandemic is ongoing.

Besides the risk of exposure to unknown COVID-19 carriers and COVID-19 positive patients, the study team could also be facing other unique challenges. Some of us have already experienced trial center personnel (in addition to trial participants) not being permitted to overcome travel restrictions and hence cannot report for duty. Still others cannot make it simply because children's schools (and crèche services) are closed. Where feasible, and where responsibilities can be fulfilled remotely, trial staff should be given the option of working from home.

Clinical Trial Investigators will also have to rework allocation of their own time for trial related activities. Times have changed dramatically in a very short time. Duties in dealing with COVID-19 pandemic will require time and priority - an emergency that would not have been factored in while agreeing to take up trials. If sufficient time is not available to cope with all the ongoing trials, a systematic decision will need to be taken on which (if any) trials to continue and which (if any) to be suspended temporarily. This will also need to be discussed with other stakeholders and officially notified as necessary.

The US FDA and National Institutes of Health have released suggestions and guidelines on how to deal with clinical trials during the ongoing COVID-19 pandemic. ${ }^{[18]}$ These include, but are not limited to, patient safety and data integrity. Many centers have stopped screening and recruitment of new patients for ongoing trials. In any case all changes thrust upon us due to the COVID-19 pandemic need to be documented in real time and reported to parties concerned - including while submitting manuscripts for publication in journals or presentation at conferences. ${ }^{[10,12]}$ US FDA has permitted the testing of trial subjects for COVID-19 positivity (if required) without the necessity of including that data in the final trial report. Also if there are changes required in the protocol or study schedule of events in order to eliminate or reduce risk to the lives or well-being of research participants (e.g., to limit exposure to COVID-19), such changes may be implemented without waiting for IRB approval or for sponsors to file the amendments with the regulatory authorities, as long as they are reported later.

Best case scenario suggests that the COVID-19 pandemic will disrupt clinical trials (and normal life) for at least 
3 months. Thereafter, what would be the circumstances are anybody's guess. What is certain is that all trials ongoing during the present pandemic shall have greater scrutiny of their data, especially with respect to symptoms associated with COVID-19 infection and pulmonary side effects. ${ }^{[6]}$

Whether the COVID-19 pandemic is shortlived or not, it will leave a lasting effect on medical progress. Some ideas will never see the light of day. Others might fall through the cracks because trials could not be completed or data collected is not robust to meet primary objective analysis. Advances anticipated from traditional health care may be pushed back. But there is always a silver lining on the darkest of clouds. New innovative solutions and technology will emerge. Trial rules may also be rewritten, with some redundant ones finally eliminated. We can hope for a brighter future while working proactively to help clinical trials face its most Serious Adverse Event, the ongoing COVID-19 pandemic.

\section{Financial support and sponsorship}

Nil.

\section{Conflicts of interest}

There are no conflicts of interest.

\section{Take home message}

Message
The ongoing global COVID-19 pandemic has become a "Serious
adverse event" for clinical trials, especially those that are ongoing
right now
Clinical Investigators need to take the initiative to play a leadership
role in protecting their patients from COVID-19 as well as
fulfilling trial related obligations
Protocol deviations, inability to comply with study schedule of
events, providing investigational drug and patient follow-up are
common challenges
Use of digital technology and social media can ensure that
compromise in data points is minimized wherever possible
Current challenges will bring the possibility of reworking how
we do trials and perhaps eliminate some of the unnecessary and
redundant legacy procedures
COVID-19: Coronavirus disease-19

\section{Purvish M Parikh', Prashant Mehta ${ }^{2}$, Krishna Mohan MVT $^{3}$, GS Bhattacharya ${ }^{4}$, K Govind Babu ${ }^{5}$}

${ }^{I}$ Department of Oncology, Shalby Cancer and Research Institute, Mumbai, Maharashtra, India, ${ }^{2}$ Department of Medical Oncology/BMT, Asian Institute of Medical Sciences, Faridabad, Haryana, India, ${ }^{3}$ Department of Medical Oncology, Basavatarakam Indo American Cancer Hospital, Hyderabad, Telangana, India, ${ }^{4}$ Department of Medical Oncology, HCG Hospitals, Bengaluru, Karnataka, India, ${ }^{5}$ Department of Medical Oncology, Salt Lake City Medical Center, Kolkata, West Bengal, India
Address for correspondence: Dr. Purvish M Parikh, Shalby Cancer and Research Institute, Mumbai, Maharashtra, India. E-mail:purvish1@gmail.com

Submitted: 30-Mar-2020

Revised: 31-Mar-2020

Accepted: 02-Apr-2020

Published: 21-Apr-2020

\section{References}

1. Available from: https://www.worldometers.info/coronavirus/. [Last accessed on 2020 Mar 30].

2. Trends, Charts, and Maps. Available from: https://clinicaltrials. gov/ct2/resources/trends. [Last accessed on 2020 Mar 16].

3. Thornton J. Clinical trials suspended in UK to prioritise COVID-19 studies and free up staff. BMJ 2020;368:m1172.

4. Available from: https://www.ncbi.nlm.nih.gov/ pubmed/?term=covid-19. [Last accessed on 2020 Mar 30].

5. McDermott MM, Newman AB. Preserving clinical trial integrity during the coronavirus pandemic. JAMA 2020. [Ahead of Print]. [Doi: 10.1001/jama.2020.4689].

6. Available from: https:/www.clinicaltrialsarena.com/comment/ COVID-19-trials-enrollment-data-analysis/. [Last accessed on 2020 Mar 30].

7. Available from: https://en.wikipedia.org/wiki/Act_of_God. [Last accessed on 2020 Mar 30].

8. Available from: https://www.nihr.ac.uk/news/dhsc-issuesguidance-on-the-impact-on-COVID-19-on-research-funded-orsupported-by-nihr/24469. [Last accessed on 2020 Mar 30].

9. Available from: https:/www.karmanos.org/karmanos/ gastrointestinal-oncology-program-karmanos. [Last accessed on 2020 Mar 30].

10. Parikh PM, Mehta P, Krishna Mohan MVT, Babu Govind. Clinical Trials facing "Serious Adverse Events" during the ongoing COVID-19 pandemic. Clinical Trials (Submitted for publication).

11. Available from: https://www.auckland.ac.nz/en/ news/2020/03/26/waking-up-in-lockdown.html. [Last accessed on 2020 Mar 30].

12. Parikh P, Mehta P, Bansal S, Aggarwal S, Patel A, Batra A, et al. Protecting healthcare professionals and workers (other than COVID-19 management facilities) from contamination during COVID-19 pandemic. Indian J Med Sci 2019. (In press).

13. Available from: https://twitter.com/IJMPOofficial/status/1240544 162576924678?s=19. [Last accessed on 2020 Mar 30].

14. Available from: http://aiihph.gov.in/minerva-mortality-in-indiaestablished-through-verbal-autopsy/. [Last accessed on $2020 \mathrm{Mar}$ $30]$.

15. Available from: https://www.mohfw.gov.in/pdf/Telemedicine.pdf. [Last accessed on 2020 Mar 30].

16. MHRA. Managing Clinical Trials During Coronavirus (COVID-19); 19 March, 2020. Available from: https://www. gov.uk/guidance/managing-clinical-trials-during-coronavirusCOVID-19. [Last accessed on 2020 Mar 30].

17. Available from: https://www.nrgoncology.org/. [Last accessed on 2020 Mar 30]. 
18. FDA Guidance on Conduct of Clinical Trials of Medical Products During COVID-19 Pandemic: Guidance for Industry, Investigators, and Institutional Review Boards. (Version March 27, 2020). US Food and Drug Administration; March, 2020 Available from: https:/www.fda.gov/regulatory-information/ search-fda-guidance-documents/fda-guidance-conduct-clinicaltrials-medical-products-during-COVID-19-pandemic. [Last accessed on 2020 Mar 30].

19. Available from: https://www.medicare.gov/medicare-coronavirus. [Last accessed on 2020 Mar 30].

20. Available from: https://www.marketwatch.com/story/drugmakers-on-shaky-ground-as-clinical-trials-are-postponed-anddrug-launches-delayed-amid-pandemic-2020-03-27. accessed on 2020 Mar 30].
This is an open access journal, and articles are distributed under the terms of the Creative Commons Attribution-NonCommercial-ShareAlike 4.0 License, which allows others to remix, tweak, and build upon the work non-commercially, as long as appropriate credit is given and the new creations are licensed under the identical terms.

For reprints contact: WKHLRPMedknow reprints@wolterskluwer.com

\begin{tabular}{|l|l|}
\hline \multicolumn{2}{|c|}{ Access this article online } \\
\hline Quick Response Code: & Website: \\
& www.ijmpo.org \\
\cline { 2 - 2 } & Dol: \\
\hline
\end{tabular}

How to cite this article: Parikh PM, Mehta P, Krishna Mohan MVT, Bhattacharya GS, Govind Babu K. "Serious Adverse Events" faced by clinical trials: Impact of the COVID-19 pandemic. Indian J Med Paediatr Oncol 2020;41:127-31. 